\title{
Quality indicators of stroke in Estonia
}

\author{
Janika Kõrv ${ }^{1}$, Andrus Kreis², Riina Vibo'
}

1 Department of Neurology and Neurosurgery, Institute of Clinical Medicine, University of Tartu, Tartu, Estonia

2 Department of Neurology, North Estonia Medical Centre, Tallinn, Estonia

\section{Background}

Appropriate management is crucial for reducing the burden of stroke.

Measurement of the quality of stroke care as a part of a process of quality improvement is important..

\section{Methods}

Quality indicators program has been launched in cooperation of the University of Tartu and

Estonian National Health Insurance Fund (NHIF) in 2013. The aim of the project is to develop standards for assessment and improvement of health services in Estonia.

As a part of the program, 5 stroke indicators were developed. The cases of acute stroke (ICD 10163 and I61) were identified retrospectively from the NHIF database.

An acute stroke case was defined when the patient had a bill for acute stroke services within 30 days of admission.

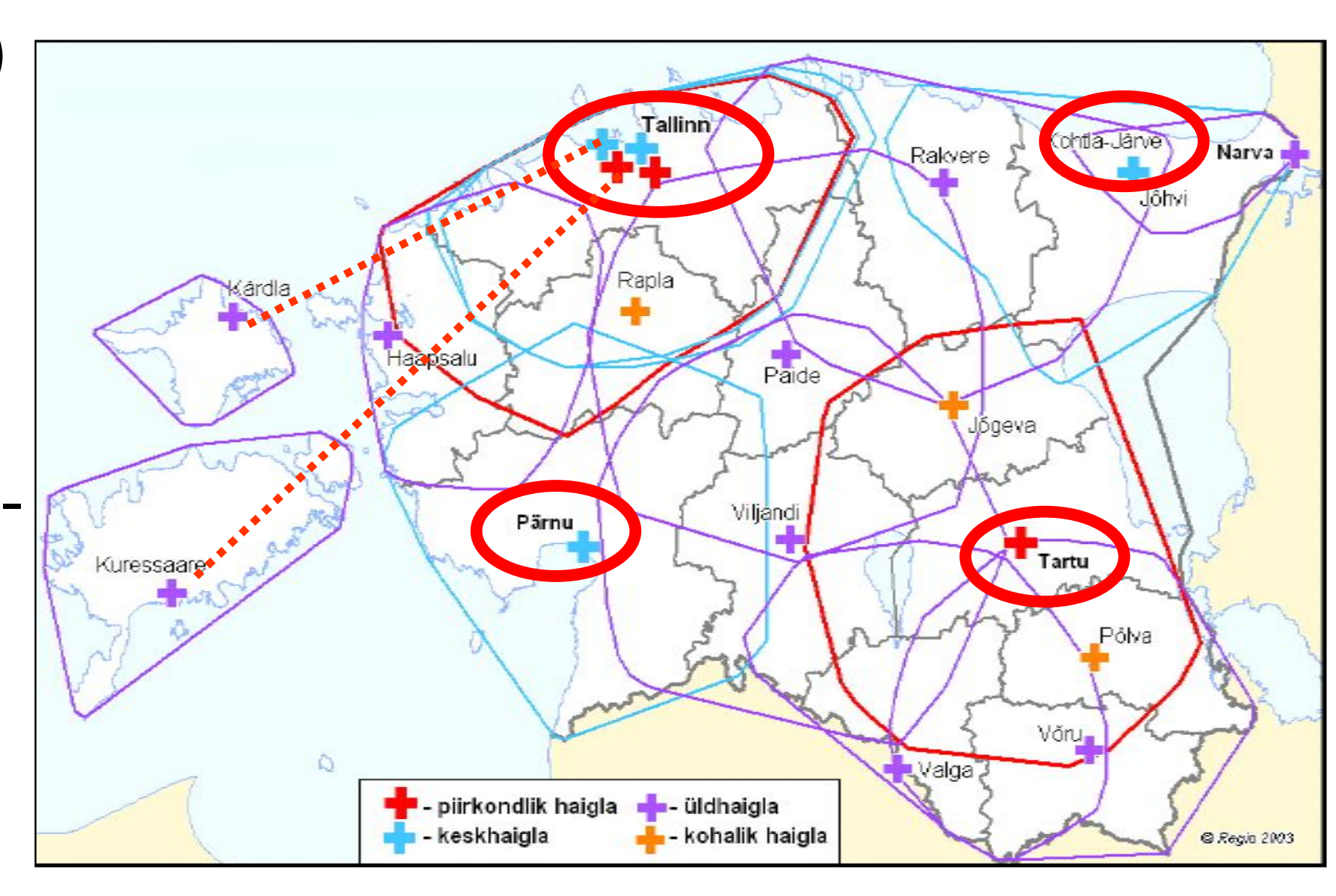

Figure. 1. Stroke Unit network
The cases were linked with the Death Registry and Medication Prescription Database. The results for 2015 and 2016 are presented. The population of Estonia is 1319133.

Annually, 4500 new strokes are diagnosed.

There are 6 hospitals with Stroke Units and 12 general hospitals (Figure 1)

\section{Results}

1. Proportion of patients managed in central or regional hospitals (24/7 stroke ready hospitals with acute Stroke Units) (Figure 2.)

Total numbers of acute strokes hospitalized:

$2015-3699$

Ischemic strokes $89 \%$

$2016-3677$

Ischemic strokes $90 \%$

A total, $77 \%$ of patients were managed in Stroke Units in 2015 and 2016 (2830 and 2834 patients, respectively).

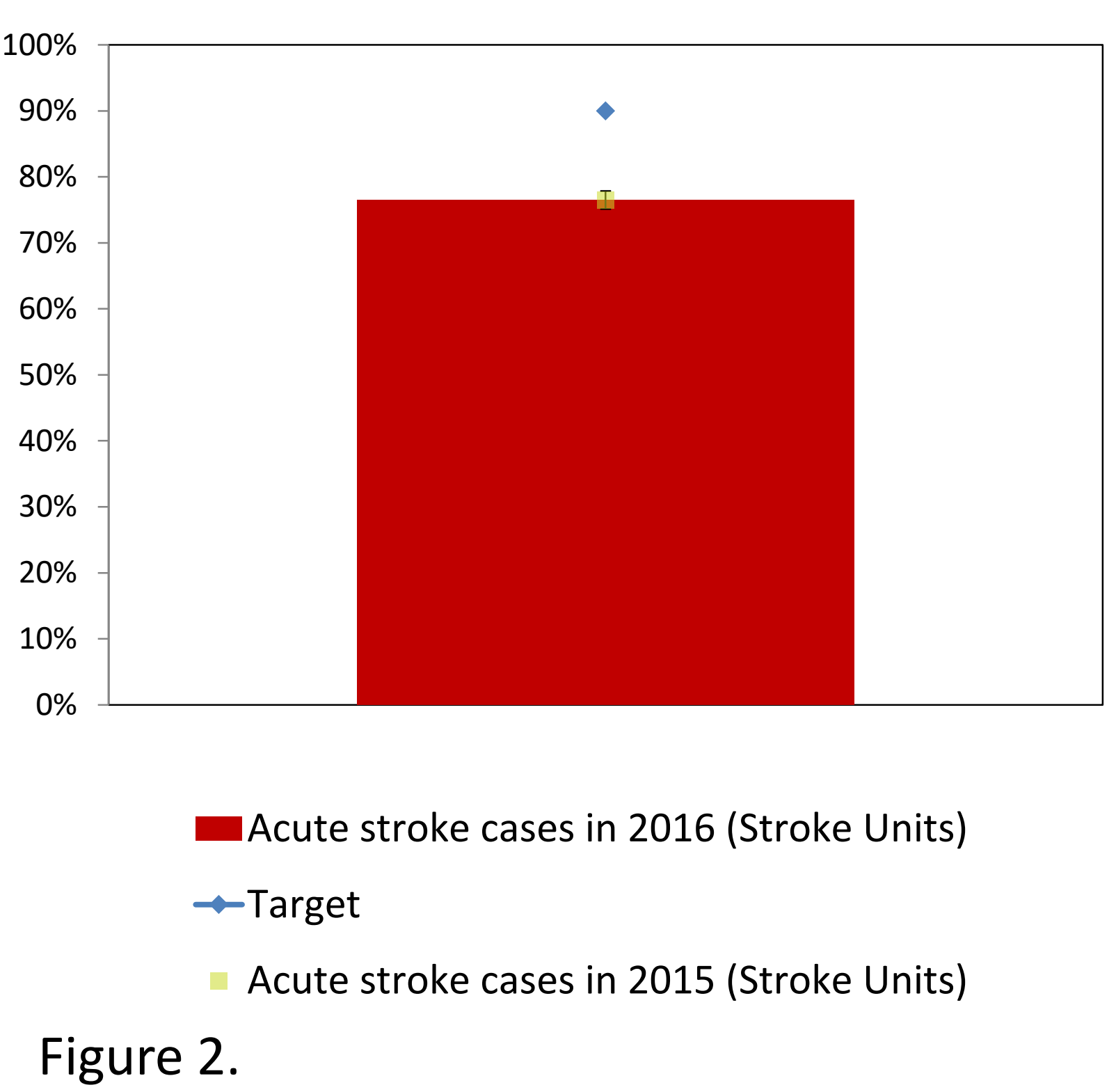

2. Proportion of patients who received recanalization therapy (iv thrombolysis or endovascular treatment) (Figure 3.)

Of all ischemic strokes $16.4 \%(95 \% \mathrm{C}$ $15.2-17.7 \%)$ and $18.2 \%(95 \% \mathrm{Cl} 16.9$ 19.6) were treated with iv rtPA in 2015 and 2016, respectively.

Endovascular treatment was not included into the calculations for 2015 and 2016

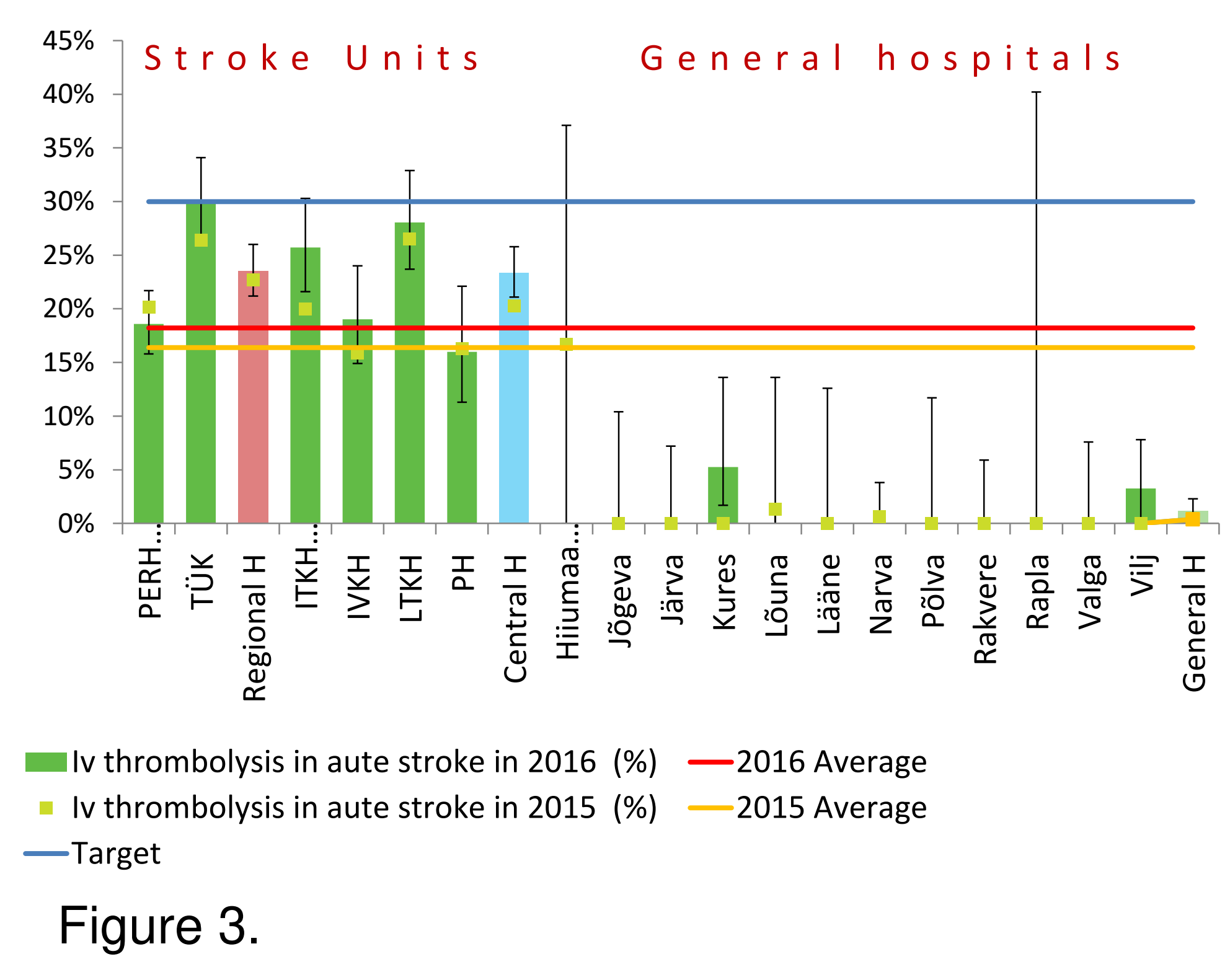

3. Proportion of patients with ischemic stroke and atrial fibrillation who have prescription for oral anticoagulant treatment 12 months +1 day after acute stroke (Figure 4.)

At 12 months after admission with ischemic stroke, $48 \%$ (95\% Cl 45.5-51.1\%) of patients with concomitant atrial fibrillation in 2015 and $52 \%(48.7-54.7 \%)$ in 2016 had an active prescription for an oral anticoagulant.

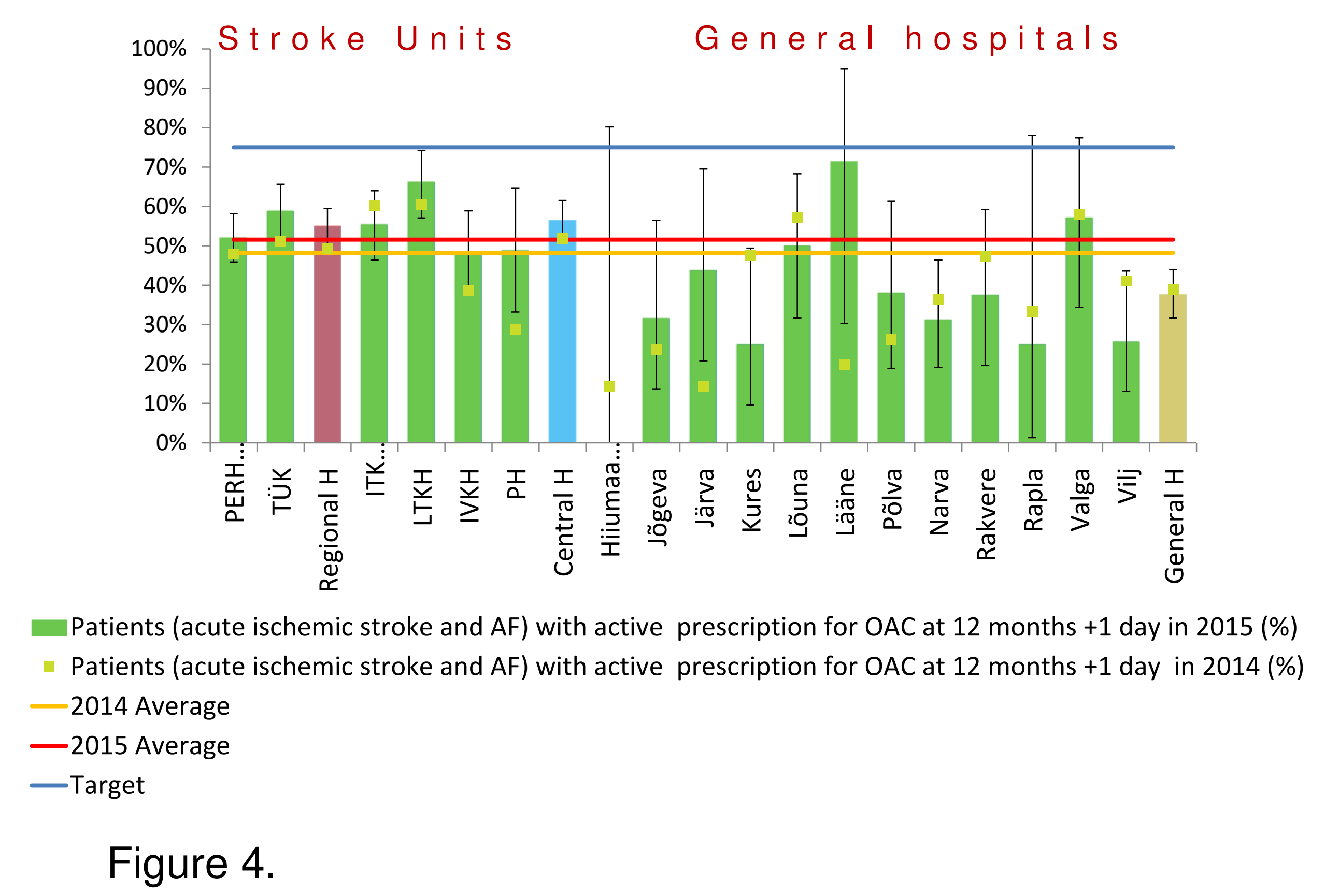

4. Proportion of patients having received rehabilitation services within 30 days onset of acute stroke (Figure 5.)

During first 30 days $68 \%$ (95\% Cl 66.2-69.2) of patients received rehabilitation services in 2015 , and $62 \%(95 \% \mathrm{Cl}$ $60.2-63.4 \%)$ in 2016 Inpatients and outpatients services were included. Inpatients rehabilitation:

Stroke Units: $45 \%$

General Hospitals: $38 \%$

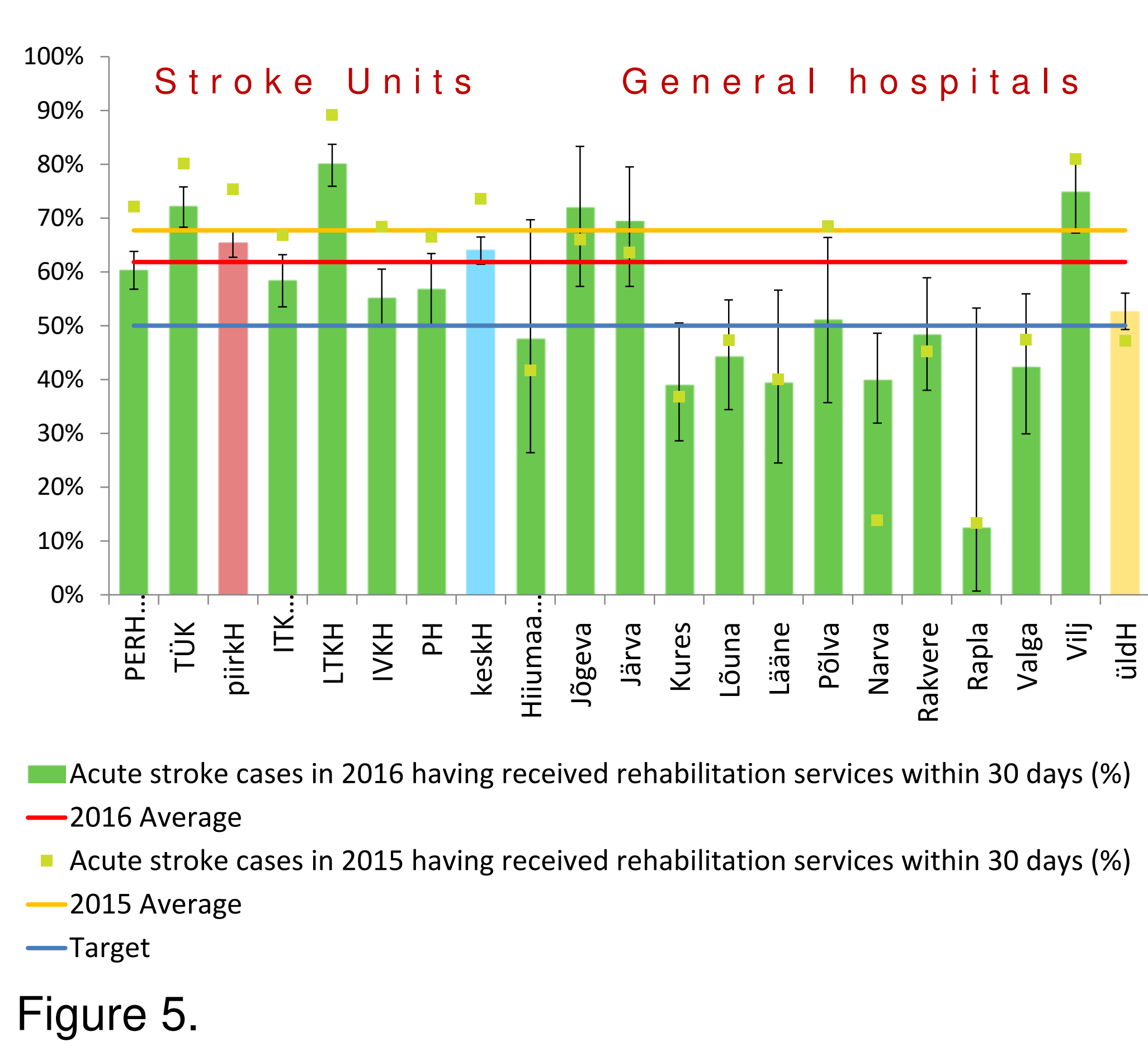

5. Proportion of patients having died within 30 days after an acute stroke (Figure 6.)

The 30-day's case fatality rate was $21 \%$ ( $95 \% \mathrm{Cl} 19.9-22.6 \%)$ in 2015 and $20 \%(18.2-20.9 \%)$ in 2016.

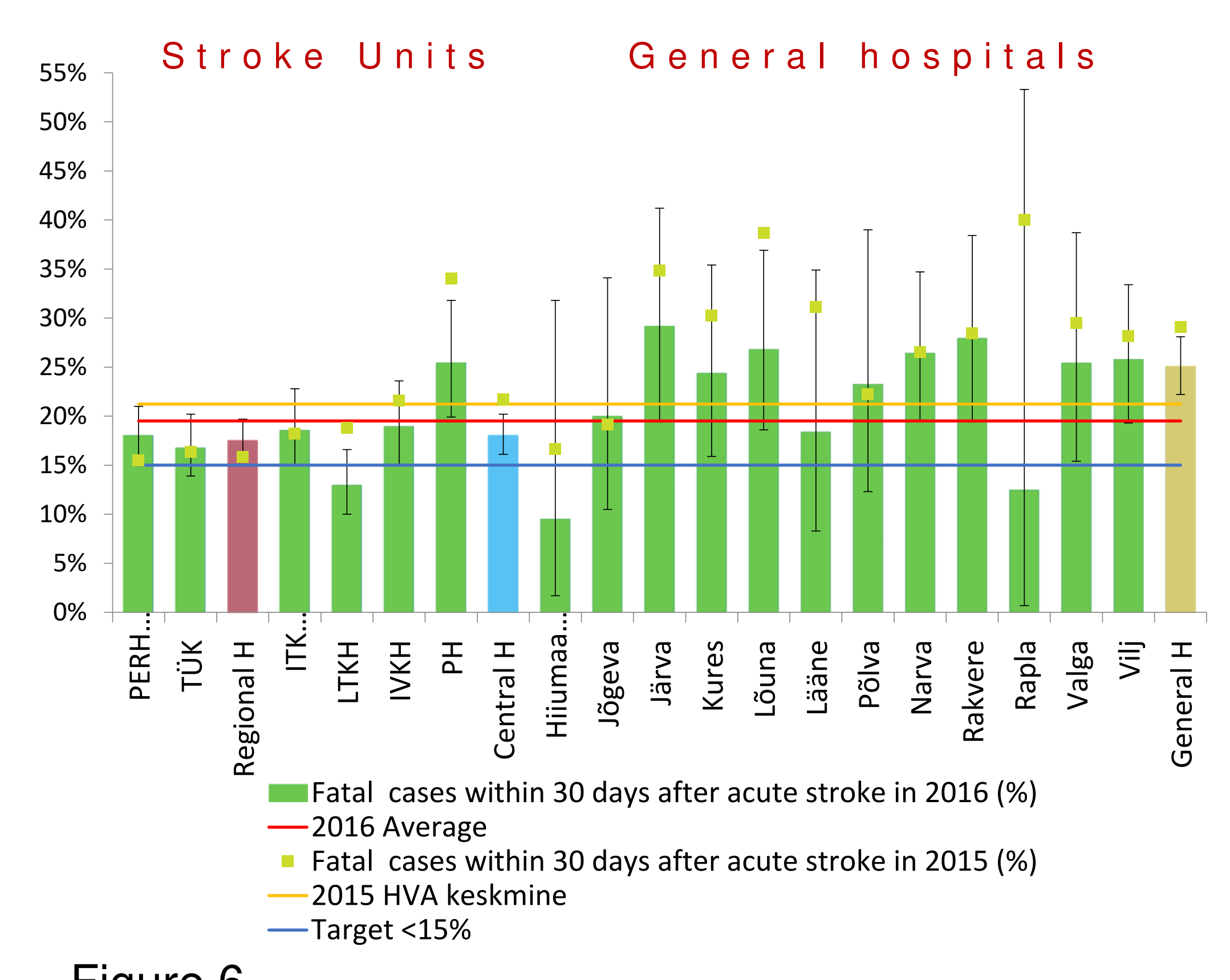

Figure 6

\section{Conclusions}

Although the indicators were somewhat behind targets, the acute management of stroke is comparable or better compared to several Western European countries. Better results in Stroke Units

Improvement in availability of iv rtPA

Problems:

Inequalities of stroke care (distant areas/islands vs big cities)

Lack of national stroke program 